\title{
Implementing the features 3D Multipatch for the management of territorial infrastructures
}

\author{
M. Caprioli, B. Figorito, A. Scognamiglio \& E. Tarantino \\ Polytechnic University of Bari, Italy
}

\begin{abstract}
The 3D geographic information has now become fundamental in the analysis and prevention of many environmental problems. For some application fields the simple 3D data visualization is required, but the 3D complex objects can be considered as a great aid in territorial analysis connected to specific areas, for instance the management of territorial infrastructures. In order to meet such requirements, the latest generation of CAD software has improved the editing and the photorealistic visualization of objects for mechanics and industrial fields, whereas GIS software, with the use of georeferenced points, lines and polygons, had emphatically developed tools for the spatial analysis of objects. So far, with visualization tools based on simple extrusions, both the technologies do not permit structuring of 3D topological models and visualization of complex entities. In literature there are substantially object-oriented and topology-oriented models. The first addresses the visualization, topology and spatial relations derived from the object's structure, while in the second, connected to analysis, relations are explicitly defined between one object and its neighbours.

With this study the 3D cartographic data processing of road networks for the realization of a prototype Infrastructure Management System was evaluated, in order to allow spatial processing in 3D GIS environments, also divulged by web. Particular attention was paid to the potentiality of the CAD-GIS tandem using the feature type Multipatch, in order to investigate the real possibility of using this methodology in many territorial application fields. The Multipatch permits one to encapsulate complex objects in a compact way and also to add textures on surfaces, providing a real objects visualization. Such a technique is still not used because commercial GIS software does not have specific tools to create feature $3 D$ Multipatch. The created objects were next visualized to verify the effective possibility of thematic queries.
\end{abstract}

Keywords: GIS 3D, CAD, Multipatch, road infrastructures. 


\section{Introduction}

All organizations from all sectors (public, private and not-for-profit) face the possibility of disruptive events that have impacts ranging from short-lived maintenance interventions to the very destruction of territorial infrastructures [4].

During the prediction and prevention studies finalized to emergency response it is preliminarily necessary to evaluate the strategic function and the vulnerability of the territorial infrastructures system useful to support protection activities. These circumstances determine the necessity of using GIS (Geographical Information System) not only for the great amount of data storage and management but above all as a useful instrument for phenomenon analysis and prevision, in order to predispose every action of surveying and planning. Modern techniques of Real Time GIS represent a great potentiality for the optimised management both for surveying and for first aid operation [12].

An irreplaceable source of information is the third dimension of territorial elements. In many interactive computer graphics applications, maps in three dimensions are central to enabling the exploration, presentation and manipulation of geographical data. 3D maps can be used by GIS specialists and as tools for presentation of spatial data to not-experts [9]. Advanced graphical libraries such as OpenGL and $V R M L$ enable effective modeling by rendering the third geographical dimension [14]. All the above representations only permit the user to view map layers as a single entity rather than being able to visualize the layers as a combined overlay representation and as individual entities in the same way that is possible with 2D GIS [5].

The structuring of 3D topological models and the visualization with thematic queries of complex entities in still not permitted in CAD and GIS technologies. In literature there are substantially object-oriented and topology-oriented models. In the first one, addressed to visualization, topology and spatial relations derive from the object structure, while in the second one, connected to analysis, relations are explicitly defined between one object and its neighbours [16].

All the 3D vectors data models share the set of geometric primitives, structured in different way for every specific application $[2,6,11,13,15]$.

The common components of any model are points, lines and solids.

The point is the most diffused in GIS environment, even if in real world there are a few entities that have not area or volume. The point is easily visualized in a three-dimensional space and is the basis for many spatial representations.

The line is a set of points connected as single one-dimensional primitive and, as the points, it is an abstraction of real objects. In the 3D space the lines are used to schematise for example cables, pipes, linear elements, without visualization of the height and the diameter or the thickness of objects.

The polygon defines a surface with common attributes and it is a twodimensional primitive constituted by one or more closed lines. It is used to represent planes, surfaces or all entities that have a two-dimensional extension without thickness. Polygons are utilized also to create 2,5 D data: the TIN is the classical example of a polygons collection with a surface and without thickness, but with a variable height. 
The solid is a primitive constituted by a set of closed polygonal faces. This is the true 3D primitive that could represent all the entities if it was easier implemented in GIS software. The definition of the order of border points in a solid is very complex and hard to implement in a geodatabase.

Also the model proposed by the "Intesa GIS" between the Italian State and Regions is not oriented to visualization and to analysis of 3D objects. The hybrid mode for $3 \mathrm{D}$ representations, through the union of a $2 \mathrm{D}$ surface with a $3 \mathrm{D}$ ring (border of an object), is not suitable to conventional data processing of the next generation GIS [1].

On the contrary, a model strongly oriented to representation of threedimensional objects in urban contexts is the CityGML, widely investigated by public and private research groups [8]. This cartographic model uses five levels of detail to represent objects and permits the insertion of texture, by using 3D virtual models aimed to sophisticated analyses in different application fields as simulations, urban data mining, facility management and also thematic complex queries. Moreover, it is implemented as application scheme for the international standard Geography Markup Language 3 (GML3). Nowadays, the implementation costs of CityGML, even if it guarantees the creation of a real 3D GIS, are still expensive for an immediate applicability.

The GML3 could represent one of the most innovative prospects towards the integration of complex and georeferenced geometries, but the instructions suggested by the Open Geospatial Consortium (OGC), in order to define the structure of the information, the metadata, the types of geographical objects, the geometric and topological relations of the entities, are hardly implemented in practical applications because of their complexity.

In the light of the Italian and international experiences in 3D GIS context, this work proposes the evaluation of the power of CAD software to manipulate threedimensional data and the properties of the Multipatch feature type in shapefile format to represent complex objects in GIS environment.

\section{Multipatch procedures for three-dimensional objects construction}

The building of Multipatches allows to encapsulate complex objects in a compact way and to add also textures on surfaces [3], providing a real objects visualization. This instrument is not yet utilized among GIS community because commercial softwares have not specific tools for the creation of 3D Multipatch features.

The Multipatch is constituted of a combination of three-dimensional surfaces (Figure 1), represented by the following geometries [7].

- Triangle Strip. It is a strip of triangles linked among them, in which every vertexes (after the first two) determines a new triangle. A new triangle is always formed by connecting the new vertex with its two immediate predecessors.

- Triangle Fan. It is a linked fan of triangles, where every vertex (after the first two) completes a new triangle. A new triangle is always formed 
by connecting the new vertex with its immediate predecessor and the first vertex of the part.

- Outer Ring. It is the outer ring of a polygon.

- Inner Ring. It is a hole of a polygon.

- First Ring. It is the first ring of an unspecified polygon.

- Ring. It is a ring of an unspecified polygon.

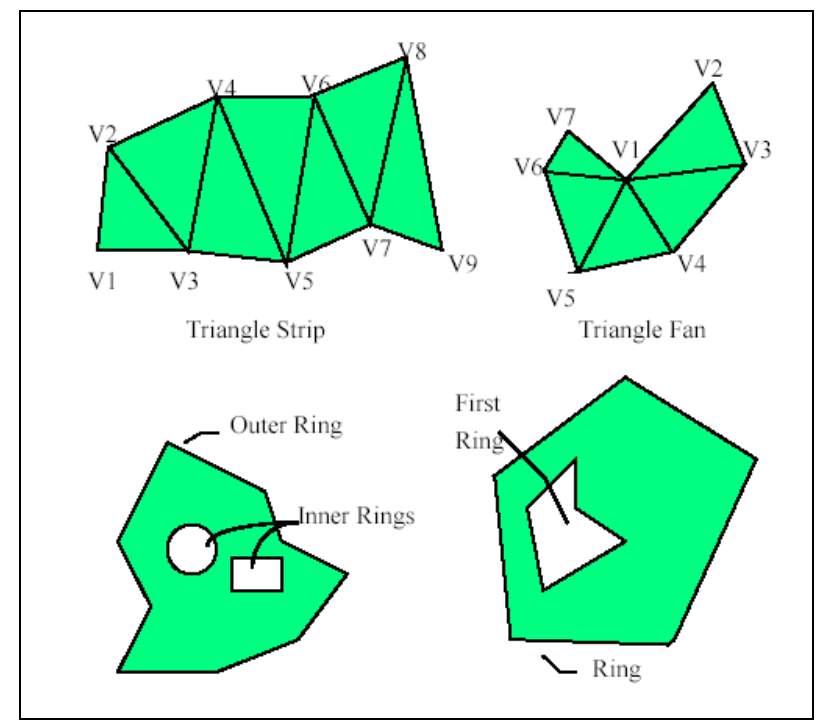

Figure 1: Geometries of the Multipatch (Source by ESRI Shapefile Technical Description, 1998).

A Multipatch is an object to all intents and purposes, similar to conventional objects (as points, lines, polygons) on shapefile format and alphanumeric attributes.

Commercial GIS software does not allow the execution of spatial queries on Multipatch. Such limit is due to the lack of 3D spatial operators. The current version of ArcGis-ArcInfo 9.2 had timidly introduced only a few functions for the editing of the Multipatch, without permitting the real development of feature type. A detailed discussion of the tested procedures with the integrated use of CAD and GIS tools follows.

\section{Methods and results}

The prototype Infrastructure Management System processed in this study is realized with the aid of AutoCAD, CAD2Shape and ArcScene - ArcInfo in order to test the possibility to use the integrated use of these technologies by many more potential users. 


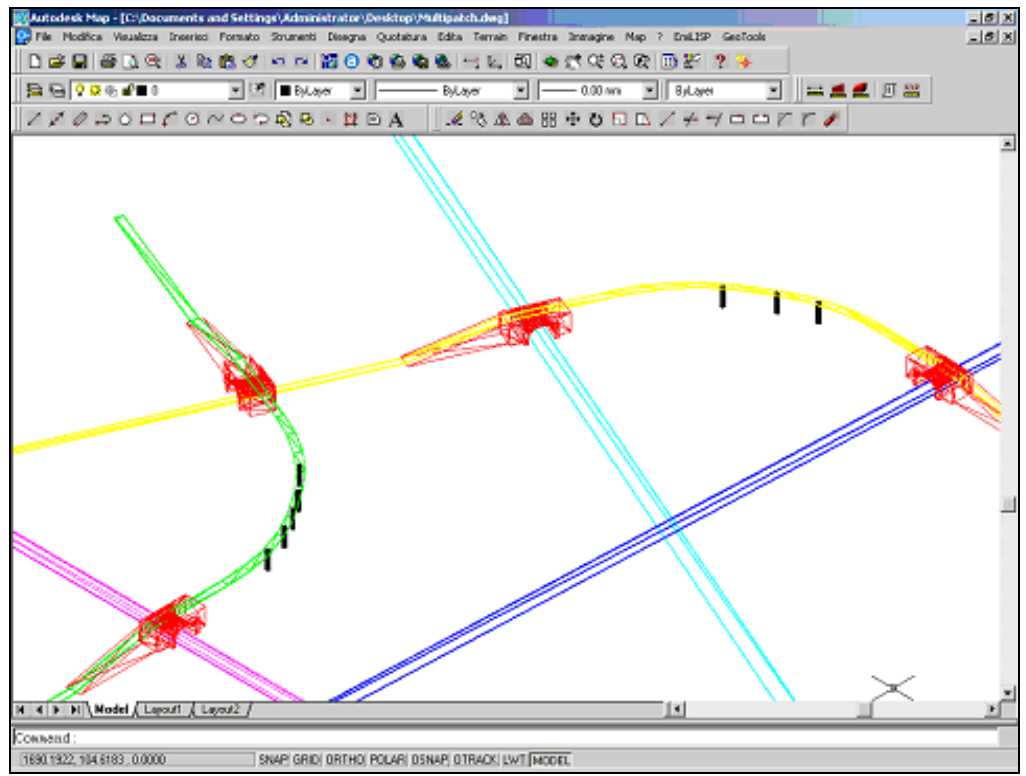

Figure 2: Example of viaducts and road networks created with CAD tools.

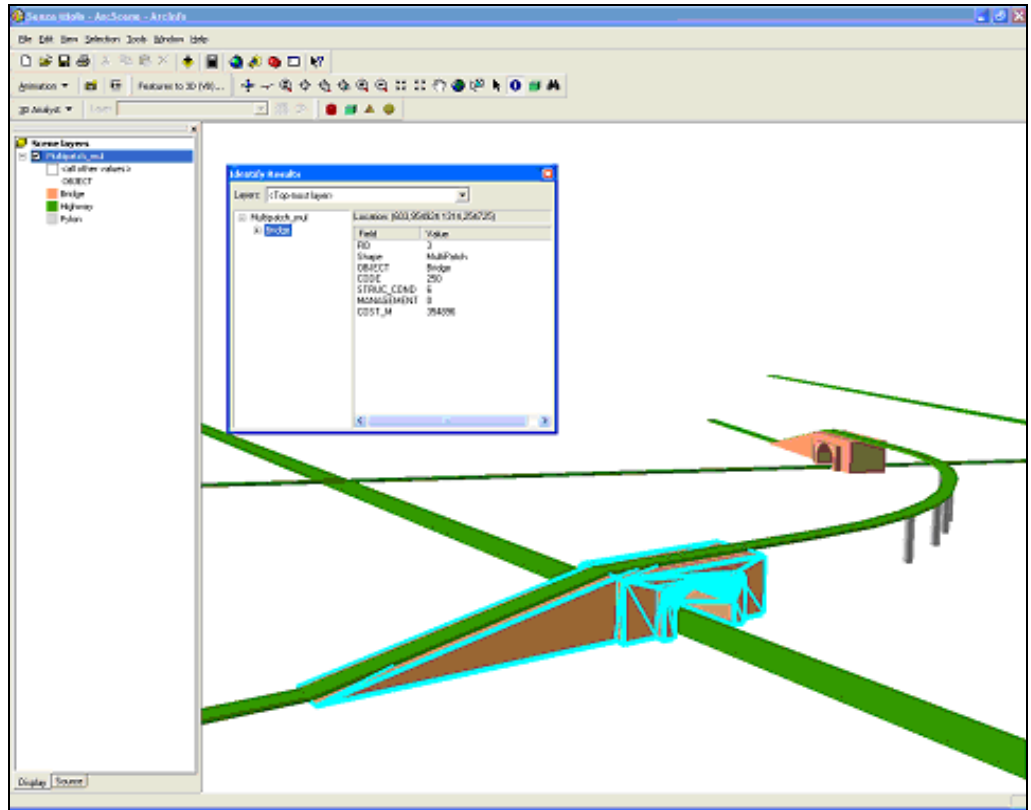

Figure 3: Alphanumeric information derived from 3D visualization on every Multipatch object. 
858 Sustainable Development and Planning III

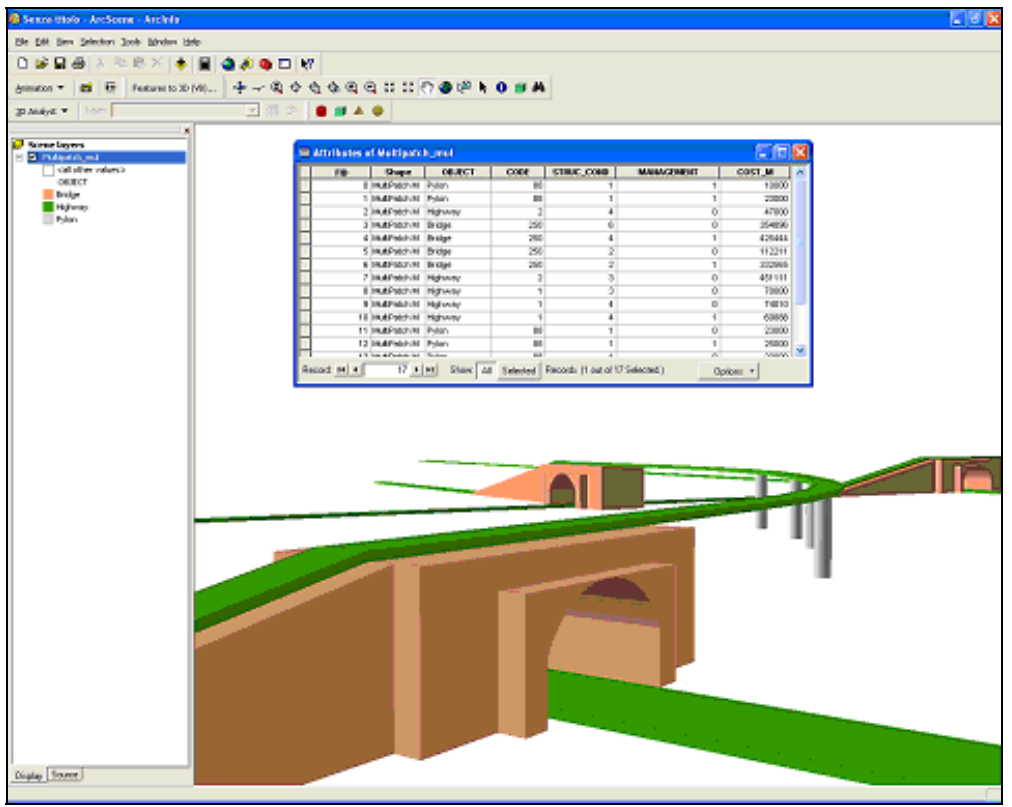

Figure 4: Report table of a prototype Infrastructure Management System.

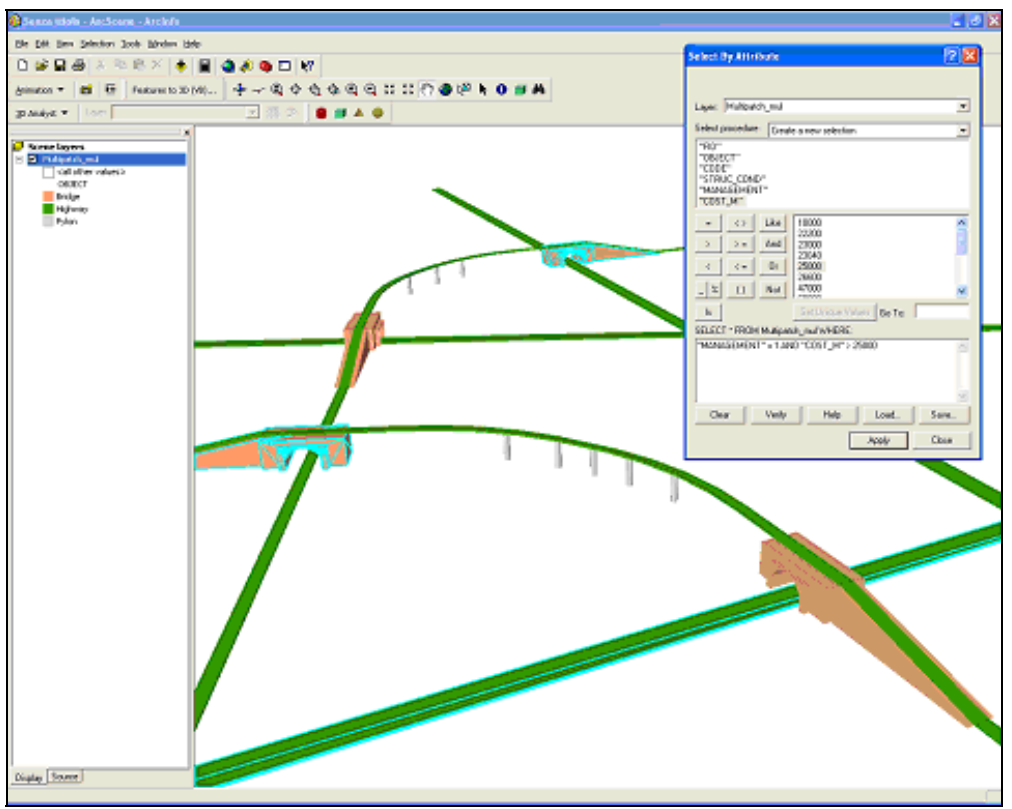

Figure 5: Execution of thematic queries on 3D objects. 
In the first phase all the objects (arcs, viaducts, connecting roads, etc.) were processed with CAD tools for the creation of three-dimensional surfaces (Figure 2).

In order to build the shapefiles with Multipatch feature type, in the second phase of the methodology we have taken advantage of the efficient conversion facilities supported by CAD2Shape. The processing times of the features depend on level of details of objects to be represented for a peculiar application field. In our case the complex entities were created using more Multipatches.

The combined use of AutoCAD and CAD2Shape tools has many advantages and could have a rapid growth among GIS community, because basic cartographic data is prevalently spread in.$d w g$ or.$d x f$ formats. Modern photogrammetric plotting, only using 3D points and polylines for object description, still does not permit the easy direct comprehension of $3 \mathrm{D}$ reality, except for a consequent time expensive editing work.

Figures 3, 4 and 5 point out the univocal representation of every object with relative thematic information derived in $3 \mathrm{D}$ interactive visualizations by means of GIS tools.

\section{Conclusion}

In this work the potentiality of the integrated use of CAD-GIS tandem using the feature type Multipatch was evaluated.

One of our key objectives in testing of procedures was to propose to GIS community the interactive querying capabilities inside of the $3 \mathrm{D}$ visualization and navigation system, in order to use them for decision making and spatial/aspatial data analysis. The implemented features have the advantage to be simple and immediately usable in current GIS softwares. In fact, the proposed methodologies, applied here to an Infrastructure Management System, could efficiently allow the retrieval and the reuse of digital cartographic archives as basic data for many application fields.

The diffusion of Multipatch objects will depend on commercial choices of CAD and GIS softwares and on the development of 3D spatial operators. The possibility of inserting textures and images on Multipatch objects will make such procedures interesting also for photo-realistic rendering of complex objects.

\section{References}

[1] Ammoscato A., Corsale R., Scianna A., Cartografia Numerica Tridimensionale per GIS e WEB-GIS: verso la fruizione virtuale, Proceedings of SIFET Conference, 14 - 17 June 2006, Taranto, Italy, (unpaginated CD-ROM).

[2] Abdul-Rahman A., The design and implementation of two and threedimensional triangular irregular network (TIN) based GIS, PhD thesis, University of Glasgow, Scotland, United Kingdom, p.250, 2000.

[3] Armit A., Multipatch and MultiObject Design Systems, Proceedings of the Royal Society London, Vol. A 321 p. 235, 1971. 
[4] Brownjohn J., Tjin S., Tan G., Tan B., Chakraboorty S. And S., A Structural Health Monitoring Paradigm for Civil Infrastructure, 1st FIG International Symposium on Engineering Surveys for Construction Works and Structural Engineering, Nottingham, United Kingdom, 28 June - 1 July 2004 (unpaginated CD-ROM).

[5] Brooks, S. and Whalley, J. L. A 2D/3D Hybrid Geographical Information System, Proceedings of ACM GRAPHITE, Dunedin, December 2005, (unpaginated CD-ROM).

[6] Coors V., 3D GIS in Networking environments, Computer, Environment and Urban Systems, 27 (4), pp. 345-357, (2003).

[7] ESRI Inc, Shapefile Technical Description An ESRI White Paper p.20-24, http://www.esri.com/library/whitepapers/pdfs/shapefile.pdf, 1998.

[8] Kolbe H., Gröger G., Plümer L., CityGML - Interoperable Access to 3D City Models, Proceedings of "International Symposium on Geoinformation for Disaster Management", 21-23 March 2005, Delft, Netherlands (CD-ROM), 2005.

[9] Maceachren, A. M., Visualization in Modern Cartography. ed. MacEachren, A. M. and Fxza, D. R. Taylor, Oxford, UK, Pergamon, 1994.

[10] Molenaar M., A formal data structure for 3D vector maps, Proceedings of EGIS'90, Vol. 2, Amsterdam, The Netherlands, pp. 770-781, 1990.

[11] Molenaar M., An Introduction to the Theory of Spatial Objects Modelling, Taylor \& Francis, London, pp. 1411-1420, 1998.

[12] Mussumeci G., Falchi U., Condorelli A., Data survey and management techniques in civil protection emergencies, Proceedings of the XXth Congress of ISPRS, Istanbul, 2004. Vol. XXXV, Part B4, pp. 1025-1030.

[13] Pilouk M., Topologic data structure for a 3D GIS, PhD thesis, ITC, 1996.

[14] Shan, J., Visualizing 3-D Geographical Data with VRML. Computer Graphics International, 108-110, 1998.

[15] Zlatanova S., 3D GIS for urban development, $\mathrm{PhD}$ thesis, ITC, The Netherlands, 222 p., 2000.

[16] Zlatanova S., Rahman, A.A., Pilouk, M., Trends in 3D GIS development, Journal of Geospatial Engineering, 2, pp. 1-10, 2002. 